\title{
BMJ Open Substance-related and addictive disorders among adults with intellectual and developmental disabilities (IDD): an Ontario population cohort study
}

\author{
Elizabeth Lin, ${ }^{1}$ Robert Balogh, ${ }^{2}$ Caitlin McGarry, ${ }^{3}$ Avra Selick, ${ }^{1}$ \\ Kristin Dobranowski, ${ }^{2}$ Andrew S Wilton, ${ }^{4}$ Yona Lunsky ${ }^{5}$
}

To cite: Lin E, Balogh R, McGarry C, et al. Substancerelated and addictive disorders among adults with intellectual and developmental disabilities (IDD): an Ontario population cohort study. BMJ Open 2016;6: 011638 .

doi:10.1136/bmjopen-2016011638

- Prepublication history for this paper is available online. To view these files please visit the journal online (http://dx.doi.org/10.1136/ bmjopen-2016-011638).

Received 22 February 2016 Revised 15 July 2016 Accepted 1 August 2016

CrossMark

For numbered affiliations see end of article.

Correspondence to

Dr Elizabeth Lin;

Elizabethbetty.lin@camh.ca

\section{ABSTRACT}

Objectives: Describe the prevalence of substancerelated and addictive disorders (SRAD) in adults with intellectual and developmental disabilities (IDD) and compare the sociodemographic and clinical characteristics of adults with IDD and SRAD to those with IDD or SRAD only.

Design: Population-based cohort study (the Health Care Access Research and Development Disabilities (H-CARDD) cohort).

Setting: All legal residents of Ontario, Canada. Participants: 66484 adults, aged 18-64, with IDD identified through linked provincial health and disability income benefits administrative data from fiscal year 2009. 96589 adults, aged 18-64, with SRAD but without IDD drawn from the provincial health administrative data.

Main outcome measures: Sociodemographic (age group, sex, neighbourhood income quintile, rurality) and clinical (psychiatric and chronic disease diagnoses, morbidity) characteristics.

Results: The prevalence of SRAD among adults with IDD was $6.4 \%$, considerably higher than many previous reports and also higher than found for adults without IDD in Ontario (3.5\%). Among those with both IDD and SRAD, the rate of psychiatric comorbidity was $78.8 \%$, and the proportion with high or very high overall morbidity was $59.5 \%$. The most common psychiatric comorbidities were anxiety disorders $(67.6 \%)$, followed by affective (44.6\%), psychotic $(35.8 \%)$ and personality disorders $(23.5 \%)$. These adults also tended to be younger and more likely to live in the poorest neighbourhoods compared with adults with IDD but no SRAD and adults with SRAD but no IDD.

Conclusions: SRAD is a significant concern for adults with IDD. It is associated with high rates of psychiatric and other comorbidities, indicating that care coordination and system navigation may be important concerns. Attention should be paid to increasing the recognition of SRAD among individuals with IDD by both healthcare and social service providers and to improving staff skills in successfully engaging those with both IDD and SRAD.

\section{Strengths and limitations of this study}

- This is a population-based study which captures individuals receiving and not receiving intellectual and developmental disabilities (IDD)-specific services and allows comparisons with non-IDD groups drawn from the same population.

- Health and disability support data were linked allowing improved identification of individuals with IDD, a group difficult to capture using single-source data.

- Administrative data were not designed with research or clinical priorities in mind meaning that some important questions could not be answered.

- There are few published validation studies for health administrative data and none, to date, for disability support administrative data due, in part, to the very heterogeneous conditions comprising IDD.

\section{INTRODUCTION}

Relatively little research has examined substance-related and addictive disorders (SRAD) in adults with intellectual and developmental disabilities (IDD). ${ }^{1}$ The prevalence studies that exist have found rates of substance use or abuse ranging between $0.5 \%$ and $2.6 \%$, which are lower than rates reported for the general population. ${ }^{2}{ }^{3}$ Individuals with IDD who use substances are more likely to be younger, male and diagnosed with milder disabilities. ${ }^{45}$ They are also at increased risk for negative consequences from substance use (eg, increased risk taking, aggression) and for becoming victims of crime and becoming involved with the justice system. ${ }^{4-8}$ A high percentage $(42-54 \%)$ are reported to have comorbid psychiatric disorders. ${ }^{49}$ These studies, however, are limited by inconsistent definitions, differences in data gathering methods, small sample sizes and 
varying inclusion/exclusion criteria $^{1}$ leading to a concern that their prevalence rates are underestimates and to calls for good population-based estimates. ${ }^{24} 1011$

Our aim is to use a large population-based cohort of adults with IDD to describe the prevalence of SRAD and to compare the sociodemographic and clinical characteristics of adults with both IDD and SRAD to other adults from the same population who only have IDD or only have SRAD.

\section{METHODS}

Data from the Health Care Access Research and Development Disabilities (H-CARDD) cohort were analysed for this study. This cohort was created by linking Ontario health administrative records (including inpatient, emergency department (ED) and physician contacts) and disability income support administrative data from fiscal 2009. These databases capture all of the healthcare users covered under Ontario's universal healthcare system as well as all recipients of provincial disability income benefits. They were linked using both probabilistic and deterministic methods (through unique encoded identifiers) and analyzed at the Institute for Clinical Evaluative Sciences (ICES); all activities related to cohort creation and this specific study were approved by the Research Ethics Boards at Sunnybrook Hospital and the Centre for Addiction and Mental Health, both in Toronto. The H-CARDD cohort is comprised of 66484 adults, aged 18-64 years, with a developmental disability (IDD). Our definition of IDD is based on the one used by our provincial government to determine disability benefit eligibility and is consistent with provincial legislation. ${ }^{12}$ It includes conditions labelled in other jurisdictions as intellectual or developmental disability. The H-CARDD cohort is, to the best of our knowledge, the largest population-based IDD cohort currently existing. The methods used to create this cohort, along with the list of included diagnostic conditions, are described elsewhere. ${ }^{13} 14$

We compared three groups: one with both IDD and SRAD (IDD-plus-SRAD), one with only IDD (IDD-only) and one with only SRAD (SRAD-only). To create these three groups, we used the H-CARDD cohort as well as a $20 \%$ sample of all Ontario adults who were aged 18-64, eligible for Ontario health benefits, but not in the H-CARDD cohort. (The latter group has been used in previous work to provide a comparative context). ${ }^{15}$ The presence of SRAD was defined as any healthcare contact within the 2 years prior to fiscal 2009 associated with a psychoactive substance-related or behavioural addiction diagnosis (essentially, F1 or F63.0 International Classification of Diseases (ICD)-10 codes or the ICD-9 or Diagnostic and Statistical Manual of Mental Disorders (DSM)-IV equivalents-for the detailed list, please contact corresponding author).

These three groups were compared on sociodemographic and clinical characteristics. Sociodemographic variables included age group, sex, rurality and neighbourhood income quintile. The measures of rurality and neighbourhood income were based on Statistics Canada definitions. Urban-rural status was derived from census subdivisions using the Statistical Area Classification of Statistics ${ }^{16}$ in which rural represents areas outside of the commuting zones of larger urban centres with a core population of 10000 or more. For the neighbourhood income measure, Ontario neighbourhoods were grouped in approximately equal-sized quintiles from poorest (quintile 1) to wealthiest (quintile 5) using 2006 census dissemination areas taking into account household size and community of residence. ${ }^{17}$

Clinical variables included measures of psychiatric disorder, five chronic physical illnesses and a comorbidity proxy. Psychiatric disorder was defined using the available diagnostic information in the health administrative data. Since the coding system varied by health data source (ICD-10 for ED and acute hospital data, broad ICD-8 categories for the physician visit data, and DSM-4 for psychiatric hospital data), our analyses used broader diagnostic categories.

The choice of the five chronic diseases was dictated by the existence of algorithms previously validated for the health administrative data we used. These were diabetes, ${ }^{18}$ hypertension, ${ }^{19}$ chronic obstructive pulmonary disorder (COPD), ${ }^{20}$ asthma $^{21}$ and congestive heart failure. $^{22}$ Comorbidity was measured using the John Hopkins Adjusted Clinical Groups (ACGs) Case-Mix System as a proxy. $^{23}$ This variable, which captures expected use of healthcare resources, has six categories (non-user, healthy user, low, moderate, high and very high). For our analyses, we examined the percentage with high or very high morbidity.

Sociodemographic characteristics were reported as crude percentages while the clinical characteristic results were adjusted for age and sex using the age-sex composition of our group of interest (IDD-plus-SRAD) as the standard. We used Cohen's d (effect size) to determine whether the observed and adjusted differences were meaningful. This statistic is independent of sample size, useful in our case because the very large numbers inflate statistical significance. It is used to represent the magnitude of difference between two groups, where 0.2, 0.5 and 0.8 are often interpreted as reflecting small, medium and large effect sizes, respectively. ${ }^{24}$ SAS V.9.4 (SAS Institute Inc. Base SAS 9.4. procedures guide: Statistical procedures. 2013) was used to generate descriptive frequencies and Wilson's ${ }^{25}$ web-based calculator to generate logit effect sizes and CIs for binary proportions. To determine meaningfulness, we used a 0.2 cut-off (equivalent to a small effect size) plus the requirement that the absolute value of the $95 \%$ CI not to go below 0.2 .

\section{RESULTS}

There were 4220 individuals in the H-CARDD cohort who met our definition of SRAD. This is equivalent to a 
prevalence rate of $6.4 \%$. Among adults without IDD, the prevalence of SRAD was $3.5 \%$.

Table 1 compares the sociodemographic characteristics of the three groups. In terms of age, sex and rurality, there were no remarkable differences between the two IDD groups ('A vs B' column), and only one small age group difference with the two IDD groups being more likely to be in the youngest age group compared with the $S R A D$-only adults (both effect sizes=0.41).

In terms of socioeconomic status (SES), over $40 \%$ of the IDD-plus-SRAD group lived in the poorest neighbourhood quintile compared with $<30 \%$ for IDD-only and $S R A D$-only adults (effect sizes $=0.2-0.4$ ). The IDD-plus-SRAD group was also less likely to live in the two wealthiest neighbourhood quintiles, particularly compared with the $S R A D$-only group (effect sizes $=-0.29$ and -0.30 ). With the exception of the poorest quintile, there were no meaningful differences between the IDD-only and SRAD-only groups based on our previously described criteria.

Larger differences were evident when the psychiatric comorbidities of the three groups were examined. In terms of unadjusted rates (not shown), over threequarters of the adults with IDD-plus-SRAD (78.8\%) had a concurrent psychiatric disorder compared with the IDD-only and SRAD-only groups $(41.4 \%$ and $51.1 \%$, respectively). The IDD-plus-SRAD adults also consistently had the highest prevalence rates when both broad categories and specific psychiatric diagnoses were considered. The most common categories were the anxiety disorders $(67.6 \%)$, followed by affective illnesses $(44.6 \%)$, and psychotic disorders $(35.8 \%)$. In terms of more specific diagnoses, $35.2 \%$ had a depressive disorder, $30.5 \%$ a schizophrenia/schizophreniform/schizoaffective disorder, $25.2 \%$ a bipolar disorder and $23.5 \%$ a personality disorder.

Adjusting these prevalence rates (and the rates for chronic illness and morbidity) by age and sex resulted in only minor changes. For example, the overall prevalence for any psychiatric disorder changed from $41.4 \%$ to $41.0 \%$ and from $51.1 \%$ to $50.6 \%$ for the IDD-only and SRAD-only groups, respectively.

Comparison of the adjusted rates (table 2) showed that the three groups differed from each other across all of our measures of psychiatric comorbidity except one (the rates of bipolar disorder for the two single-condition groups). The largest differences were between the two IDD groups: of the nine comparisons in column 'A vs B', only the 'other' comparison had an effect size below 0.8 . The IDD-plus-SRAD also differed from the SRAD-only group with all effect sizes in the medium-to-large range. Differences between the two single-condition groups were less marked with all effect sizes below the medium (0.5) threshold. With the exception of the psychotic and 'other' categories, the IDD-only adults had the lowest adjusted rates of psychiatric comorbidity of the three groups.

Similar but less marked patterns were found when the age-sex-adjusted rates of chronic illness and morbidity were examined (table 3). As with psychiatric

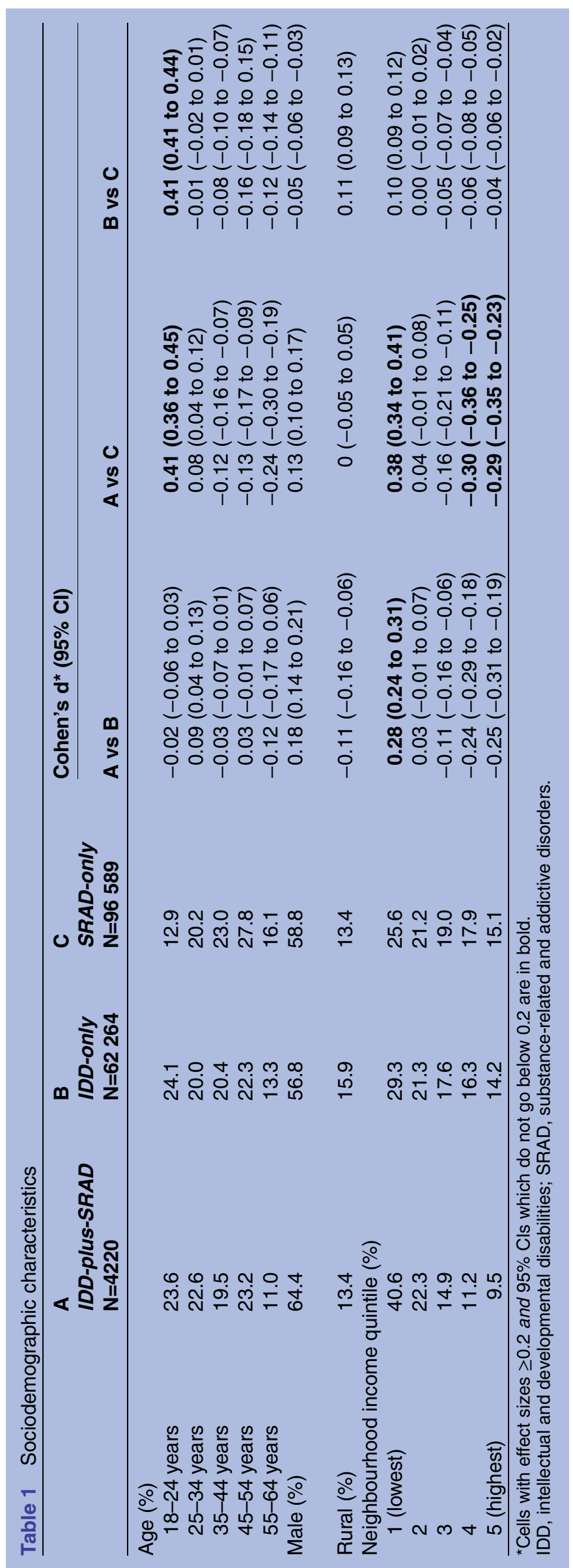


Table 2 Age-adjusted and sex-adjusted clinical characteristics: psychiatric disorders

\begin{tabular}{|c|c|c|c|c|c|c|}
\hline & \multirow{2}{*}{$\begin{array}{l}\text { A } \\
\text { IDD-plus-SRAD } \\
\mathrm{N}=4220\end{array}$} & \multirow{2}{*}{$\begin{array}{l}\mathrm{B} \\
\text { IDD-only } \\
\mathrm{N}=62264\end{array}$} & \multirow{2}{*}{$\begin{array}{l}\text { C } \\
\text { SRAD-only } \\
\mathrm{N}=96589\end{array}$} & \multicolumn{3}{|l|}{ Cohen's d* $(95 \% \mathrm{Cl})$} \\
\hline & & & & A vs $B$ & A vs C & B vs C \\
\hline \multicolumn{7}{|c|}{ Psychiatric disorders (excluding SRAD) } \\
\hline ANY & 78.8 & 41.0 & 50.6 & $0.92(0.88$ to 0.97$)$ & $0.71(0.67$ to 0.75$)$ & $-0.21(-0.23$ to -0.20$)$ \\
\hline Psychotic & 35.8 & 9.8 & 5.8 & $0.90(0.86$ to 0.94$)$ & $1.21(1.18$ to 1.25$)$ & $0.31(0.29$ to 0.33$)$ \\
\hline Schizophrenia & 30.5 & 8.5 & 4.1 & $0.86(0.82$ to 0.90$)$ & 1.28 (1.24 to 1.32$)$ & $0.43(0.40$ to 0.45$)$ \\
\hline Affective & 44.6 & 10.5 & 18.0 & 1.06 (1.03 to 1.10$)$ & 0.72 (0.68 to 0.75$)$ & $-0.35(-0.36$ to -0.33$)$ \\
\hline Depressive & 35.2 & 7.5 & 14.8 & 1.05 (1.01 to 1.09$)$ & $0.63(0.59$ to 0.66$)$ & $-0.42(-0.44$ to -0.40$)$ \\
\hline Bipolar & 25.2 & 4.6 & 6.4 & 1.07 (1.03 to 1.12$)$ & $0.88(0.84$ to 0.92$)$ & $-0.19(-0.22$ to -0.17$)$ \\
\hline Anxiety & 67.6 & 29.4 & 43.3 & 0.89 (0.85 to 0.93$)$ & 0.55 (0.52 to 0.59$)$ & $-0.33(-0.35$ to -0.32$)$ \\
\hline Personality & 23.5 & 3.3 & 4.9 & 1.21 (1.17 to 1.26$)$ & 0.98 (0.94 to 1.03$)$ & $-0.23(-0.26$ to -0.20$)$ \\
\hline Other & 13.1 & 7.5 & 4.8 & 0.34 (0.29 to 0.39$)$ & 0.60 (0.55 to 0.66$)$ & $0.26(0.24$ to 0.29$)$ \\
\hline
\end{tabular}

IDD, intellectual and developmental disabilities; SRAD, substance-related and addictive disorders.

Table 3 Age-adjusted and sex-adjusted clinical characteristics: chronic physical illness and overall morbidity

\begin{tabular}{|c|c|c|c|c|c|c|}
\hline & \multirow{2}{*}{$\begin{array}{l}\text { A } \\
\text { IDD-plus-SRAD } \\
\mathrm{N}=4220\end{array}$} & \multirow{2}{*}{$\begin{array}{l}\text { B } \\
\text { IDD-non-SRAD } \\
\text { N=62 } 264\end{array}$} & \multirow{2}{*}{$\begin{array}{l}\mathrm{C} \\
\text { SRAD-non-DD } \\
\mathrm{N}=96589\end{array}$} & \multicolumn{3}{|l|}{ Cohen's d* $(95 \% \mathrm{Cl})$} \\
\hline & & & & A vs $B$ & A vs C & B vs C \\
\hline \multicolumn{7}{|l|}{ Chronic physical illness } \\
\hline Diabetes & 12.6 & 9.8 & 6.5 & $0.16(0.10$ to 0.21$)$ & $0.40(0.35$ to 0.45$)$ & $0.25(0.23$ to 0.27$)$ \\
\hline Hypertension & 15.3 & 13.8 & 13.2 & 0.07 (0.02 to 0.11$)$ & $0.09(0.05$ to 0.14$)$ & $0.03(0.01$ to 0.04$)$ \\
\hline COPD & 13.6 & 5.2 & 8.5 & $0.58(0.53$ to 0.63$)$ & $0.29(0.24$ to 0.34$)$ & $-0.29(-0.31$ to -0.27$)$ \\
\hline Asthma & 27.3 & 16.5 & 18.0 & $0.35(0.31$ to 0.39$)$ & $0.30(0.26$ to 0.33$)$ & $-0.06(-0.07$ to -0.04$)$ \\
\hline $\begin{array}{l}\text { Congestive heart failure } \\
\text { Morbidity }\end{array}$ & 2.0 & 1.0 & 0.7 & $0.39(0.26$ to 0.51$)$ & $0.59(0.46$ to 0.71$)$ & $0.20(0.14$ to 026$)$ \\
\hline Per cent of high/very high morbidity & 59.5 & 21.6 & 43.2 & $00.92(0.89$ to 0.96$)$ & $0.36(0.33$ to 0.40$)$ & $-0.56(-0.57$ to -0.55$)$ \\
\hline
\end{tabular}

${ }^{*}$ Cells with effect sizes $\geq 0.2$ and $95 \%$ Cls which do not go below 0.2 are in bold.

COPD, chronic obstructive pulmonary disorder; IDD, intellectual and developmental disabilities; SRAD, substance-related and addictive disorders. 
comorbidity, adults with IDD-plus-SRAD consistently had the highest disease and morbidity rates of the three groups. The most common chronic illness in this group was asthma (27.3\%) followed by hypertension, COPD and diabetes (12.6-15.3\%). When the groups were compared, both IDD groups had higher rates of diabetes than the SRAD-only group with the difference between the IDD-plus-SRAD and SRAD-only groups being particularly large $(12.6 \%$ vs $6.5 \%$, effect size $=1.66)$. No difference was found between the IDD-only and SRAD-only groups for asthma, but both showed small differences compared with the IDD-plus-SRAD group (effect sizes $=0.3-0.35$ ). All three groups showed small differences from each other for COPD and congestive heart failure but no differences for hypertension. In terms of high or very high morbidity, the largest difference was between the two IDD groups $(59.5 \%$ vs $21.6 \%$, effect size $=0.92)$ with the other morbidity comparisons falling into the small and moderate effect size ranges.

\section{DISCUSSION}

\section{Principal findings}

The prevalence of SRAD among adults with IDD was $6.4 \%$, compared with $3.5 \%$ in those without IDD. Individuals with both IDD and SRAD had the highest rates of overall morbidity $(78.8 \%$, psychiatric comorbidity; $59.5 \%$, high or very high morbidity) and of specific illnesses. The most common psychiatric comorbidities were anxiety disorders $(67.6 \%)$, followed by affective (44.6\%), psychotic $(35.8 \%)$ and personality disorders (23.5\%) while the most common chronic diseases were asthma (27.3\%), hypertension (15.3\%), COPD (13.6\%) and diabetes $(12.6 \%)$. They also tended to be younger and more likely to live in the poorest neighbourhoods compared with adults with IDD-only or SRAD-only.

\section{Study strengths and weaknesses}

Our work combines several strengths when compared with other reported studies. The H-CARDD cohort is based on data which capture nearly the entire population of a single geopolitical jurisdiction. It includes individuals receiving IDD-specific social services as well as those who have only accessed health services. As such, it avoids some of the biases inherent in analysing smaller clinical samples. It also allows comparisons to groups drawn from the same population and using consistent operational definitions. These provide more detail than previous reports on how the combination of IDD and $S R A D$ differs from either condition alone. Finally, the use of linked data provides more comprehensive coverage of the IDD population compared with studies using single-source data. ${ }^{13} 26$

Balancing these strengths are some important limitations. First, like many other studies, our definition of IDD has not been externally validated, due in part to the heterogeneous group of conditions classified as IDD. There are few published validation studies of administrative data for this population. Thus far, validation studies for health administrative data exist for autism spectrum disorders ${ }^{27-29}$ and Down syndrome. ${ }^{30}$ We have found no validity research for either social services or disability support administrative data. Second, administrative data are not usually collected to serve research or even clinical purposes. Consequently, important variables such as illness severity, type of addiction, the type of care delivered, poverty or ethnicity are not typically captured, and thus important questions such as what is the quality and appropriateness of care or the effect of social factors cannot be answered. Third, despite using linked data sources, it is likely that some people with IDD or with addictions were missed. ${ }^{26}$ The most likely gaps are individuals who were diagnosed with IDD as children or adolescents but who were not recorded as having IDD in adulthood in either the health or disability income support data. ${ }^{13}$ Finally, while a strength of this study is that it captures an entire geopolitical population, the results may not be generalisable to other jurisdictions that have very different health and social service systems or IDD populations.

\section{Important differences in results}

Our prevalence for SRAD (6.4\%) is considerably higher than previously reported rates. ${ }^{1}$ It is also higher than the prevalence we report for adults without IDD $(3.5 \%)$ or what has been found in the Canadian general population $(4.4 \%) .{ }^{31}$ The mostly likely explanation for these differences is our use of linked, population-based data sources which may have captured a larger proportion of individuals with milder forms of $I D D$ who are living in the community and not necessarily accessing IDD-specific supports. ${ }^{1126}$ Researchers have suggested that this group is at greater risk for exposure to substances and the risk factors that support substance use and abuse. ${ }^{145}$

In addition, we report a prevalence of $79 \%$ for psychiatric illnesses among adults with the combination of IDD and SRAD which is higher than the $42-54 \%$ previously reported. ${ }^{1}{ }^{4}$ A closer look at other populationbased studies does not, unfortunately, provide definitive evidence on whether our higher prevalence is an overestimate, an accurate estimate (possibly because of our use of linked data) and/or a function of the definitions and methods we used. Cooper et $a l \mathrm{~s}^{2}$ study, which reported a prevalence of $41 \%$ with 'mental ill health of any type' ( $p .30$ ), used a combination of disability support records and detailed clinical assessments to define their study cohort of adults with intellectual disabilities. However, they did not look at the prevalence of mental ill health in the subset with comorbid SRAD, and it is also unclear what the impact would be of their case ascertainment methods versus ours. To the best of our knowledge, Slayter's ${ }^{9}$ study is the only populationbased study to examine rates of psychiatric comorbidity among the subset with an addiction. Their case ascertainment is more similar to ours than the one used by Cooper et al, and they report a prevalence of $54 \%$ among US Medicaid recipients. However, their study 
differs from ours in two ways: first, they only assessed 'serious mental illness' (p.53); second, they only looked at intellectual disability (ID; (mental retardation). It seems reasonable that our examination of all (and not just serious) psychiatric illnesses in a population which included both $I D$ and development disability would yield a higher prevalence rate, but what the true magnitude of that rate should be is not clear.

Like other studies, we found that the SRAD group is younger and male. ${ }^{13}$ We did not find any other studies about SES although it has been suggested that living in poverty puts individuals with IDD at risk for substance abuse. ${ }^{1}$ Finally, while it has been suggested that substance abuse impacts the physical health of individuals with $I D D,{ }^{1}$ ours is the first study to describe this association at a population level and also to compare it with individuals using substances but who do not have IDD.

\section{Implications}

Our findings support those researchers who have identified SRAD as a significant problem in this population. ${ }^{4} 1126$ More specifically, our results suggest that within the already complex IDD population, SRAD is a marker for even more complex, serious conditions as well as for a need to attend closely to engagement, intervention and, in particular, cross-sector care coordination. $^{32}$ Other researchers have commented on the need for staff training (about IDD for addictions services staff and about $S R A D$ for providers of IDD-specific care). ${ }^{1}$ Services and supports that prevent, identify, treat and manage the significant mental health and addiction problems of adults with IDD need to be developed and integrated into their programmes of care. In addition, the need to link with social services because of risk factors associated with poverty and also the increased risk of legal involvement associated with $S R A D$ have been noted. ${ }^{13}{ }^{3}$ Finally, navigational supports are critical because of the increased information processing demands on the person with IDD and their support system. These supports would serve as a starting point for coordinating across the health, mental health, housing and public health services required to adequately meet the complex needs of persons with IDD and addictions. To date, research providing evidence about what strategies improve engagement, intervention and system coordination for this population is still in its infancy, and our findings emphasise the need to continue the pursuit of this kind of work.

\author{
Author affiliations \\ ${ }^{1}$ Provincial System Support Program, Centre for Addiction and Mental Health, \\ Toronto, Ontario, Canada \\ ${ }^{2}$ Faculty of Health Sciences, University of Ontario Institute of Technology, \\ Oshawa, Ontario, Canada \\ ${ }^{3}$ Cancer Care Ontario, Toronto, Ontario, Canada \\ ${ }^{4}$ Institute for Clinical Evaluative Sciences, Toronto, Ontario, Canada \\ ${ }^{5}$ Underserved Populations Program, Centre for Addiction and Mental Health, \\ Toronto, Ontario, Canada
}

Contributors EL, RB and YL were the primary creators of the concept and design of this study. The analytic approach was created by EL, RB and ASW and carried out by ASW. EL was responsible for creating the first draft and integrating all co-author input. All co-authors were involved in the acquisition of the data, contributed substantively to the interpretation of the results and to draft revisions, and have approved the final version of the manuscript.

Funding This work was supported by the Province of Ontario through their research grants programme; a Partnerships in Health Systems Improvement grant (PHE \#103973) from the Canadian Institutes of Health Research (CIHR); and the Institute for Clinical Evaluative Sciences (ICES), which is funded by an annual grant from the Ontario Ministry of Health and Lon-Term Care (MOHLTC). Parts of this material are based on data and information compiled and provided by the Canadian Institute for Health Information $(\mathrm{CIHI})$.

Disclaimer The analyses, conclusions, opinions and statements expressed here are those of the authors, and not necessarily those of the Province, $\mathrm{CIHR}$, or the data providers. No endorsement by the Province, CIHR, the Institute for Clinical Evaluative Sciences (ICES), or $\mathrm{CIHI}$ is intended or should be inferred.

Competing interests None declared.

Ethics approval Sunnybrook Hospital REB; Centre for Addiction and Mental Health REB.

Provenance and peer review Not commissioned; externally peer reviewed.

Data sharing statement No additional data are available.

Open Access This is an Open Access article distributed in accordance with the Creative Commons Attribution Non Commercial (CC BY-NC 4.0) license, which permits others to distribute, remix, adapt, build upon this work noncommercially, and license their derivative works on different terms, provided the original work is properly cited and the use is non-commercial. See: http:// creativecommons.org/licenses/by-nc/4.0/

\section{REFERENCES}

1. Taggart L, Chaplin E. Substance misuse. In: Tsakanikos E, McCarthy J. eds. Handbook of psychopathology in intellectual disability: research, practice, and policy. Autism and child psychopathology series. New York: Springer Science+Business Media, 2014:205-23.

2. Cooper SA, Smiley E, Morrison J, et al. Mental ill-health in adults with intellectual disabilities: prevalence and associated factors. Br J Psychiatry 2007;190:27-35.

3. Chaplin E, Gilvarry C, Tsakanikos E. Recreational substance use patterns and co-morbid psychopathology in adults with intellectual disability. Res Dev Disabil 2011;32:2981-6.

4. Carroll Chapman SL, Wu LT. Substance abuse among individuals with intellectual disabilities. Res Dev Disabil 2012;33:1147-56.

5. To WT, Neirynck S, Vanderplasschen W, et al. Substance use and misuse in persons with intellectual disabilities (ID): results of a survey in ID and addiction services in Flanders. Res Dev Disabil 2014;35:1-9.

6. Taggart L, McLaughlin D, Quinn B, et al. An exploration of substance misuse in people with intellectual disabilities. J Intellect Disabil Res 2006;50(Pt 8):588-97.

7. McGillivray JA, Moore MR. Substance use by offenders with mild intellectual disability. J Intellect Dev Disabil 2001;26:297-310.

8. Petersilia J. Invisible victims. Hum Rights 2000;27:9-12.

9. Slayter EM. Disparities in access to substance abuse treatment among people with intellectual disabilities and serious mental illness. Health Soc Work 2010;35:49-59.

10. Cocco K, Harper DC. Substance abuse in people with mental retardation: assessing potential problem areas. Ment Health Asp Dev Disabil 2002;5:101-8.

11. Van Duijvenbode N, VanDerNagel JEL, Didden R, et al. Substance use disorders in individuals with mild to borderline intellectual disability: current status and future directions. Res Dev Disabil 2015;38:319-28.

12. Government of Ontario. Services and supports to promote the social inclusion of persons with Developmental Disabilities Act, 2008. Toronto ON. 2010.

13. Lin E, Balogh R, Isaacs B, et al. Strengths and limitations of health and disability support administrative databases for population-based health research in intellectual and developmental disabilities. J Policy Pract Intellect Disabil 2014;11:235-44. 
14. Lin E, Balogh RS, Cobigo V, et al. Using administrative health data to identify individuals with intellectual and developmental disabilities: a comparison of algorithms. J Intellect Disabil Res 2013;57:462-77.

15. Lunsky Y, Klein-Geltink JE, Yates EA, eds. Atlas on the primary care of adults with developmental disabilities in Ontario. Toronto ON: Institute for Clinical Evaluative Sciences and Centre for Addiction and Mental Health, 2013

16. Statistics Canada. Standard Geographical Classification (SGC). Volume I. The classification. Ottawa ON, 2007. http://www.statcan. gc.ca/pub/12-571-x/12-571-x2006001-eng.pdf

17. Wilkins R, Khan S. PCCF+Version $5 \mathrm{H}$ User's Guide. Automated geographic coding based on the Statistics Canada Postal Code Conversion files, including postal codes through October 2010 [Internet]. 2010. http://abacus.library.ubc.ca/jspui/bitstream/10573/ 42833/3/msword.pccf5j.pdf

18. Hux JE, Flintoft V, Ivis F, et al. Diabetes in Ontario: determination of prevalence and incidence using a validated administrative data algorithm. Diabetes Care 2002;25:512-16.

19. Tu JV, Naylor DC, Austin P. Temporal changes in the outcomes of acute myocardial infarction in Ontario, 1992-1996. Can Med Assoc $J$ 1999;161:1257-61.

20. Gershon AS, Wang C, Guan J, et al. Identifying individuals with physician diagnosed COPD in health administrative databases. $J$ Chronic Obstr Pulm Dis 2009;6:388-94.

21. Gershon AS, Wang C, Guan J, et al. Identifying patients with physician-diagnosed asthma in health administrative databases. Can Respir J 2009;16:183-8.

22. Schultz SE, Rothwell DM, Chen Z, et al. Identifying cases of congestive heart failure from administrative data: a validation study using primary care patient records. Chronic Dis Inj Can 2013;33:160-6.
23. Weiner JP, Abrams C. The Johns Hopkins ACG System Technical Reference Guide, version 9.0. The Johns Hopkins Bloomberg School of Public health. 2009.

24. Cohen J. A power primer. Psychol Bull 1992;112:155-9.

25. Wilson DB. Effect Size Calculator (cited 7 May 2015). http://www. campbellcollaboration.org/escalc/html/EffectSizeCalculator-SMD9. php

26. VanDerNagel JEL, Kiewik M, Postel MG, et al. Capture recapture estimation of the prevalence of mild intellectual disability and substance use disorder. Res Dev Disabil 2014;35:808-13.

27. Pelly L, Vardy C, Fernandez B, et al. Incidence and cohort prevalence for autism spectrum disorders in the Avalon Peninsula, Newfoundland and Labrador. CMAJ Open 2015;3(3): E276-80.

28. Dodds L, Spencer A, Shea S, et al. Validity of autism diagnoses using administrative health data. Chronic Dis Can 2009;29:102-7.

29. Van Naarden Braun K, Pettygrove S, Daniels J, et al. Evaluation of a methodology for a collaborative multiple source surveillance network for autism spectrum disorders-autism and developmental disabilities monitoring network, 14 sites, United States, 2002. MMWR Surveill Summ 2007;56:29-40.

30. Jensen KM, Cooke CR, Davis MM. Fidelity of administrative data when researching Down syndrome. Med Care 2013;00:1-6.

31. Pearson C, Janz T, Ali J. Health at a glance: mental and substance use disorders in Canada. Ottawa ON: 2013. http://www.statcan.gc. ca/pub/82-624-x/2013001/article/11855-eng.pdf

32. Slayter E, Steenrod Shelley A. Addressing alcohol and drug addiction among people with mental retardation in nonaddiction settings: a need for cross-system collaboration. J Soc Work Pract Addict 2009:9:71-90. 